\section{LES ALPES INDUSTRIELLES}

Sous ce titre, a paru, le i 5 décembre dernier, dans la Revue de Paris, un très intéressant article de M. Houllevigue; reprenant à larges traits les travaux et les constatations de M. R. Tavernier, les condensant en quelques pages élégantes et lumineuses, M. Houllevigue en a composé un tableau très remarquable dont la lecture doit intéresser tous ceux qui s'occupent de houille blanche.

La tendance et les conclusions de l'auteur aboutissent à ceci : une législation intelligente doit s'adapter à la situation économique nouvelle créée par la science, et la puissance publique n'a pas le droit de se désintéresser de ce qui va advenir; faut-il laisser se former, aux dépens de la fortune publique, une nouvelle forme de propriété privée? Les chutes d'eatu sont le bien de tous, parce qu'elles sont utiles à tous; les puissances hydrauliques du pays appartiennent à la communauté. La solution indiquée serait la concession des forces motrices par l'Etat, selon des dispositions analogues à celles proposées le 6 juillet 1900 par le Gouvernement, mais avec indication des correctifs suivants: les concessions d'eau accordées aux industriels devront avoir une durée suffisante pour leur permettre de se rémunérer largement de leurs efforts; elles les assureront contre toutes les tracasseries, qu'elles viennent des particuliers ou de l'Etat; enfin tout devra être fait pour les encourager à multiplicr leurs installations et à aménager leurs chutes d'une façon rationnelle; mais aussi, à l'expiration de leurs concessions, les services publics bénéficieront de cette inépuisable puissance des rivières de France, et pourront l'attribuer aux œuvres d'intérêt général, en louant l'excédent aux industries particulières.

Une prochaine solution s'impose, dit en terminant M. Houllevigue, nous devons souhaiter que, soucieuse des intérêts particuliers, elle affirme cependant sans équivoque l'intérêt et le droit de la communauté.

Evidemment la loi doit viser à ce que la communauté, l'ensemble du pays, retire tout le profit possible de la mise en valeur de nos chutes d'eau; là-dessus aucun désaccord n'est possible, et ce serait une méprise de croire que ceux qui ont réuni le Congrès de septembre dernier à Grenoble l'ont réuni pour chercher à faire prédominer des intérêts particuliers contre les intérêts généraux; ce Congrès a été ouvert largement; tous les systèmes, toutes les opinions ont été invités à s'y manifester, et s'y sont manifestés. De leur côté aussi, les nombreuses Chambres de Commerce qui, depuis moins de deux ans, ont étudié le projet de loi sur les usines hydrauliques se sont préoccupées, dans leurs délibérations et leurs conclusions, beaucoup plus des intérêts généraux que des intérêts particuliers.

Pourquoi chercher à susciter entre ces deux groupes d'intérêts un conflit qui n'existe pas? L'un n'est-il paș la somme de tous les autres? Et si on désire voir les Alpes devenir industrielles, il convient d'éviter avant tout ce qui risquerait de les rendre plutôt administratives ou électorales.

¿a mise en valeur des chutes d'eau doit compter avec deux prémisses que $M$. Houllevigue a parfaitement fait ressortir : l'irrégularité du débit des cours, d'eau et les. dépenses élevées de l'aménagement. En imposant à nos. cours d'eau de montagne cet étiage ou cet hivernage périodiyue, parfois prolongé, où la puissance se réduit au tiers, au dixième de sa valeur moyenne, la nature réduit par cela même les services publics à une portion congrue. Cés services ne peuvent pas chômer pendant des semaines entières. Ils doivent donc borner leurs vues tout au plus à l'utilisation de l'énergie hydraulique d'étiage, c'est-à-dire de la moindre portion de la puissance annuelle disponible dans nos cours d'eau. Et comme il n'en coûte pas beaucoup plus, en aménageant une chute d'eau pour le débit minimum, d'élargir les ouvrages pour pouvoir utiliser l'énergie moyenne de la rivière, c'est la seule industrie privée que la nature elle-même semble évoquer pour la mise en valeur. Ce sont des particuliers surtout qui pourront s'ingénier à tirer parti de cet énorme sous-produit des eaux moyennes, sous-produit massif par rapport au produit marchand qui est l'énergie des eaux basses. Et en utilisant cette ressource que la nature a prodiguée à notre pays, ces particuliers contribueront à la prospérité générale, ils vivifieront des contrées inactives, ils ménageront nos bassins houillers et économiseront nos importations de combustibles étrangers (200 millions par an environ). Lorsqu'ils auront mis en valeur les chutes d'eau, le kilowat s'offrira sur le marché en concurence avec le charbon. C'est alors que les services publics pourront acheter leurs kilowatts, se les faire livrer là où ils en ont besoin, lorsqu'ils en auront besoin; déjà les offres abondent, la concurrence est ouverte. Si les services publics devaient c.ıpter eux-mêmes leur énergie, ils seraient rivés à quelques lieux de production, leur établissement serait alourdi par de grosses immobilisations supplémentaires; les chemins de fer n'ont pas commis l'erreur d'acheter des concessions de mines pour s'y alimenter. Si telle ou telle chute d'eau était marquée en rouge pour être destinée et affectée à un service public, ce serait renoncer presque fatalement à tout le bénéfice de l'énergie de ses eaux moyennes? Celui qui en douterait ferait bien de se rendre compte de ce qui se passe en réalité dans certains pays dont la législation n'est pas enviable, au moins sous ce rapport.

Il est vrai qu'en Suisse, en avril s 89 , la société Freiland a proposé de déclarer fédérales toutes les forces bydrau. liques non encore utilisées; mais les cantons consultés par le Gouvernement répondirent à l'unanimité que ce n'était pas aux dépens du bien-être général que la valeur nouvelle de la force hydraulique était abandonnée à la spéculation et. à l'industrie privées. Et après une enquête approfondie le gouvernement fédéral ne jugea pas opportun de donner suite à la proposition de la société Freiland.

En Italie toutes les eaux sont publiques, l'Etat en dispose par des concessions renouvelables, par conséquent quasi perpétuelles, qui ne sont autre chose qu'un contrat de cession d'une certaine quantité d'eau motrice (Voir La Houille Blanche du 15 juin 1902), et ceux-là même qui réclament aujourd'hui la nationalisation complète, comme M. le professeur F. S. Nitti de Naples, sont les premiers à 
s'élever contre les tendances des services publics à se réserver la primauté sur l'usage des chutes d'eau.

Il est trop tard aujourd'hui, et de plus de dix ans, pour qu'on puisse déplacer en France l'assiette du droit d'usage des eaux sans violenter de nombreuses situations acquises, sans froisser des intérêts considérables et respectables. Une loi précise nous manque; elle est restée à l'étude durant presque tout le siècle dernier; elle a été proposée par le gouvernement en I 880 ; tronquée, peut-être pour amorcer les nouvelles tendances, elle a abouti à la loi du 8 avril 1898 qui s'arrête avant le titre des eaux utiles, mais la discussion de cette loi a clairement montré les intentions du législateur d'alors, et la jurisprudence a progressivement depuis un siècle déterminé et assis les bases de notre droit sur l'usage des eaux. Supprimer les tracasseries des particuliers serait un progres, la mise en valeur de notre richesse y gagnerait, en même temps que l'intérèt général et les services publics, mais ne nous faisons pas l'illusion de croire qu'on pourrait éviter les tracasseries de l'Etat. On sait très bien aujourd'hui, on voit tous les jours comment les collectivités considèrent et traitent leurs concessionnaires en matière de mines, de gaz, de tramways, de services subventionnés, etc. En général l'Etat pose comme premiers principes de décliner toute responsabilité vis-à-vis du public, et de ne répondre de ses actes que devant la juridiction administrative.

C'est en Italie, pays dépourvu de combustible fossile, et où la houille blanche est si merveilleusement distribuée, que l'utilisation des forces hydrauliques à la traction des voies ferrées a été le mieux étudiée. Én décembre 1897 , une commission composce de fonctionnaires appartenant au Royal Inspectorat général des voies ferrées et aux compagnies de chemins de fer y fut cluargée d'étudier l'application de la traction électrique aux chemins de fer à trafic limité. Ses travaux ont été publies à Rome en I 899 . Ils ont abouti à l'organisation d'une séric d'essais qui sont en cours à l'heure actuelle. Plus récemment, une nouvelle commission administrative était instituée par un décret du 2o juillet 1901, pour l'étude du réseau complémentaire. Son rapport, daté du 18 juin 1902 , a été imprimé à. Rome (tipografia dell'unione cooperativa editrice via di Forta Salaria 23-A). Il contient un paragraphe IV, sur l'utilisation des forces hydrauliques pour l'adoption éventuelle de la traction électrique; dont 'j'ai essayé de donner la traduction après cette note, parce qu'il me paraît contenir le résumé le plus actuel de l'opinion la mieux informée sur le parti que les services publics peuvent tirer de la houille blanche pour leur traction. On s'illusionne beaucoup trop souvent sur l'étendue de ce parti.

Concluons donc que l'intérêt de la collectivité doit passer en effet avant tout, mais que cet intérêt ne consiste pas nécessairement à réserver à l'Etat le monopole ou même l'attribution des forces hydrauliques; il consiste surtout à ce que les forces hydrauliques soient bien employées selon les services qu'elles peuvent rendre à l'activité générale du pays. Comme les services publics ne peuvent en utiliser que la plus faible puissance, ce n'est pas à eux que la nature parait avoir dévolu la charge d'aménager les chutes d'eau, c'est plutôt à l'initiative privée, dont le jeu est le meilleur ressort pour une bonne mise en valeur. L'industrie privée, la plus grosse consommatrice de l'énergie, saura trouver les capitaux nécessaires aux créations utiles, et alimenter librement les services publics tout en s'ingéniant à tirer le meilleur parti de cette richesse irrégulière, en améliorant les conditions dans lesquelles la nature nous la présente.

$$
\begin{gathered}
\text { Ch. Pivat. } \\
\text { Ancien Ingénietur des Ponts el Chaussées, } \\
\text { Mấtre de Forges à Allevard. }
\end{gathered}
$$

\section{ANNEXE}

Traduction du Rapport de la Commission rorale Italienne pour l'Etude des Chemins de fer complémentaires. (is juin 1902).

IV. - Sur l'utilisation des forces hydrauliques pour l'adoption éventuelle de la traction électrique. - Appliquer la traction électrique à quelqu'une des voies ferrées complémentaires, en utilisant et transformant l'énergie de nos sources naturelles, tel est le problème qu'ont étudié sur place plusieurs des sous-commissions en même temps qu'elles étudiaient les tracés; et, tandis que certaine a à peine indiqué, par exemple, pour les lignes Coni-Virtimille et Fano-Santa Barbara qu'on pourra, dans l'avenir, faire état de l'énergie hydraulique de la Roïa ou du Métauro, d'autres ont écarté cette utilisation pour des raisons dont limportance mérite d'être mise ici en relief.

Vers la fin de 1897 , on le sait, fut nommée une commission avec mandat d'étudier l'application de la traction electrique aux chemins de fer à faible trafic. Cette commission a déterminé, dans son rapport qui recueillit l'adhésion des gens compétents, les cas dans lesquels l'application de la traction électrique pouvait particulièrement convenir.

Notre commission a fait état des résultats de ces études, spécialement en s'occupant des lignes Lagonegro-SpezzanoCastrovillari, Aulla-Lucca et Spilimbergo-Gemona; la première se développe le long des vallées du Noce, du Mercure et du Coscile, où l'on trouve des rivières portant un débit considérable, même dans leurs plus basses eaux; on pourrait les utiliser sans grande difficulté en créant les chutes voulues le long de leur cours; pour la seconde, Aulla-Lucca, le Serchio, l'Aulella, la Magra peuvent fournir une quantité d'énergie motrice certainement surabundante pour ses besoins du trafic; sur la troisième, Spilimbergo Gemona, on trouve les grandes forces hydrauliques du Tagliamento et de ses affluents, pour lesquelles des concessions sont déjà demandées.

Mais la "Commission a observé en thèse générale que, dans l'état actuel de l'électrotechnique, on devait exclure l'application de la traction électrique de celles des lignes dont le trafic n'est ni intense, ni continu, et qui doivent être éxploitées par un petit nombre de paires de trains lourds. Le combustible représente dans les dépenses d'exploitation un élément minine, de telle sorte que cette faible économie compenserait à peine la lourde charge des grandes installations que rendrait nécessaires la transformation du système de traction. En effet, c'est lévidence, la transformation devrait être faite de manière à pouvoir réaliser toute la puissance du système "ordinaire, c'est-à-dire de manière à pouvoir tractionner par moment des trains lourds, et on immobiliserait ainsi des fortes hydrauliques dont on ne 
lirerait en moyenne qu'un parti assez restreint; en sorte que la traction électrique convient à une exploitation par des trains légers et assez fréquents; par contre, pour des trains lourds et rares, la traction à vapeur assure le service avec une élasticité et une ampleur incomparablement meilleures.

Pas davantage l'emploi des accumulateurs ne donne de solution, soit parce que leur rendement est assez faible et qu'il en résulte une dépense d'exploitation élevée, soit parce que, si on veut les faire assez importants pour éviter des générateurs trop puissants, on s'engage dans de trop grosses déperses d'établissement.

En outre, dans certains cas on ne saurait conseiller, et il ne serait pas spécialement avantageux d'adopter les pentes et les courbes plus accentuées, dont s'accommoderait la traction électrique.

La Commission a enfin examiné si, paraventure, les industries locales pourraient en même temps s'accommoder de l'énergie affecrée à l'exploitation de la voie ferrée, en l'utilisant, soit pendant les longs intervalles entre les trains, soit aux moments où la charge sur la ligne serait assez faible. Mais elle a reconnu que cette compensation répondrait mal aux exigences, soit d'une industrie privée quelconque, soit d'un service public de chemin de fer et que, sans aucun doute, de graves inconvénients nâेtraient de cette permutation irrégulière dans l'emploi de la même source d'énergie motrice par deux consommateurs ayant en vue des utilisations si diverses. Peut-être, et la Commission ne l'avance qu'avec beaucoup de réserve, cette utilisation pourrait-elle se réaliser, mais seulement dans le cas où l'exploitant de la ligne se trouverait être lui-même propriétaire de l'usine où s'emploierait l'énergie transformée.

Pour ces motifs, la Commission a conclu que les riches sources de la Toscane, de la Calabre et du Frioul soient laissées à la disposition de telles industries qui viendraient à se créer ou à se développer par suite de la création de nouvelles voies ferrées; et que ces industries, puisant dans notre précieux patrimoine d'eaux publiques la force morrice nécessaire à leur établissement ou à leur épanouissement, apporteraient aux futures lignes ferrées un important contingent de trafic.

Il serait superflu de dire que ces mêmes raisons qui ont conduit la Commission à ne pas adopter la traction électrique sur ces lignes à faible trafic, l'ont elles-mêmes amenée à se déterminer en faveur de la traction électrique sur la nouvelle ligne "direttisima» Rome-Naples, pour laquelle on prévoit un irafic intense, continu et à grande vitesse.

$\mathbf{v}$

\section{TRAMWAYS SANS RAILS}

\section{A PRISE DE COURANT AÉRIENNE}

\section{Description du système.}

Considérations générales. - La grande facilité d'application, la souplesse et la sécurité de la traction électrique ont amené rapidement la transformation de la traction animale et mécanique sur rails, des tramways de presque toutes les grandes villes, en traction électrique.

Favorisée par les besoins croissants des moyens de transports rapides de notre époque, cette transformation, de même que la création de nouveaux réseaux, s'est opérée avec une rapidité aussi surprenante qu'imprudente dans certains cas. C'est, en effet, par suite de cette précipitation que nombre d'entreprises ont périclité, l'importance des capitaux engagés étant disproportionnée aux ressources du trafic trop restreint et matériellement insuffisantes pour rémunérer ces capitaux.

Il ressort clairement de ces faits que la traction sur rails, dans laquelle les frais de premier établissement sont toujours très élevés, rend impossible la mise en. exécution d'un certain nombre de lignes où le trafic est trop faible, ou du moins trop incertain, pour rémunérer les capitaux, payer les frais d'entretien, d'amortissement et d'exploitation.

Il était donc intéressant de chercher un mode de traction pouvant convenir, au double point de vue technique et financier, à ces lignes à faible trafic.

A première vue, il semblerait que l'emploi d'automobiles portant eux-mêmes - ou elles-mêmes - leur source d'énergie, fut tout indiqué. Mais les nombreux essais qui ont été tentés jusqu'à ce jour prouvent que les batteries d'accumulateurs, les machines à vapeur et les moteurs à pétrole, n'ont pas toute l'élasticité voulue et sont d'un poids trop élevé (en moyenne 8300 kilos pour 24 voyageurs) pour vaincre d'une manière satislaisante les difficultés dé la traction des poids lourds sur route.

Le fonctionnement toujours quelque peu incertain du mécanisme de ces véhicules et leur entretien coûteux rendent techniquement et financièrement impraticable un service public de voitures routières. Point n'est besoin, d'ailleurs, de citer à l'appui de cette opinion de nombreux échecs dont nul n'est sans avoir entendu parler.

Voitures électriques routières. - Bien différentes'sont lés conditions de marche des automobiles routières qui reçoivent leur énergie d'une station centrale à l'aide d'unè canalisation électrique aérienne. Dans ce cas, on peut en effet réduire à son minimum le poids mort du véhicule et. c'est là ce qui fait la supériorité de ce mode de traction. Il est hors de doute que l'électricité est l'agent le plus apte à la réalisation des conditions auxquelles les voitures pour les transports en commun ou la remorque des poids lourds doivent satisfaire, c'est-à-dire: légèreté, élasticité de marche, facilité de manœuvre, frais d'entretien minima du mécanisme moteur.

Est-il nécessaire de rappeler qu'au surplus les moteurs électriques n'engendrent pas de trépidations, qu'ils ont l'avantage, très grand pour des voitures routières, de pouvoir être logés dans carters hermétiquement clos, et enfin qu'ils ne demandent pas une surveillance constante : il suffit de veiller de temps en temps à ce que le graissage se fasse bien. Nous aurions mauvaise grâce à insister ici sur les avantagés de l'application des électromoteurs à la traction : une plume plus autorisée que la nôtre ( $\mathrm{I}$ ) se charge de montrer les progrès et l'avenir des chemins de fer électriques. Nous voudrions seulement, dans un ordre d'idées infiniment plus modestes, démontrer que les tramways électriques sans rails

(r) Voir les articles de M.Barbillion, sur la traction électrique aux grandes vitesses et aux grandes distances, dars La Houille Blanitie, $\mathrm{n}^{0 \mathrm{H}} 4$ et 8, rgor et I, 1903 . 